ACCEPTED MANUSCRIPT

\title{
A flow-intrinsic trigger for capturing reconfigurations in buoyancy-driven flows in automated PIV
}

To cite this article before publication: Michael Mommert et al 2019 Meas. Sci. Technol. in press https://doi.org/10.1088/1361-6501/ab0619

\section{Manuscript version: Accepted Manuscript}

Accepted Manuscript is "the version of the article accepted for publication including all changes made as a result of the peer review process, and which may also include the addition to the article by IOP Publishing of a header, an article ID, a cover sheet and/or an 'Accepted

Manuscript' watermark, but excluding any other editing, typesetting or other changes made by IOP Publishing and/or its licensors"

This Accepted Manuscript is @ 2019 IOP Publishing Ltd.

During the embargo period (the 12 month period from the publication of the Version of Record of this article), the Accepted Manuscript is fully protected by copyright and cannot be reused or reposted elsewhere.

As the Version of Record of this article is going to be / has been published on a subscription basis, this Accepted Manuscript is available for reuse under a CC BY-NC-ND 3.0 licence after the 12 month embargo period.

After the embargo period, everyone is permitted to use copy and redistribute this article for non-commercial purposes only, provided that they adhere to all the terms of the licence https://creativecommons.org/licences/by-nc-nd/3.0

Although reasonable endeavours have been taken to obtain all necessary permissions from third parties to include their copyrighted content within this article, their full citation and copyright line may not be present in this Accepted Manuscript version. Before using any content from this article, please refer to the Version of Record on IOPscience once published for full citation and copyright details, as permissions will likely be required. All third party content is fully copyright protected, unless specifically stated otherwise in the figure caption in the Version of Record.

View the article online for updates and enhancements. 


\title{
A flow-intrinsic trigger for capturing reconfigurations in buoyancy-driven flows in automated PIV
}

\author{
Michael Mommert ${ }^{1,2}$, Daniel Schiepel ${ }^{1}$, Daniel Schmeling ${ }^{1}$, \\ Claus Wagner ${ }^{1,2}$ \\ ${ }^{1}$ Institute of Aerodynamics and Flow Technology, German Aerospace Center (DLR), \\ Göttingen, Germany \\ ${ }^{2}$ Institute of Thermodynamics and Fluid Mechanics, Technische Universität \\ Ilmenau, Ilmenau, Germany \\ E-mail: michael.mommert@dlr.de \\ $* * * * * * * 2019$
}

\begin{abstract}
In order to acquire flow fields of rare reconfiguration events in buoyancydriven flows, an automated flow-intrinsic trigger method for 3D particle image velocimetry (PIV) was developed. The trigger condition for initiating PIV is based on continuous multi-probe temperature measurements. The autonomously started PIV was realized with LED illumination and a controlled helium-filled soap bubble generator. The measured velocity fields reveal the transition between two different flow states in a mixed convection sample as proof of concept for this method. It is further proposed to consider the time series of all temperature probes for the trigger condition using Proper Orthogonal Decomposition (POD). It is demonstrated that the use of this POD-based trigger condition allows to detect flow events much earlier than the trigger based on single raw temperature signals.
\end{abstract}

PACS numbers: $47.20,47.80$

Keywords: conditional trigger, PIV, rare flow events, automated measurement, buoyancy-driven flows

Submitted to: Meas. Sci. Technol. 


\section{Flow-intrinsic trigger for PIV}

\section{Introduction}

Rare flow-state reconfiguration events are typical for buoyancy-driven flows, such as Rayleigh-Bénard convection [1,2] and mixed convection [3]. They inhere a typical duration and occur stochastically [1] with a frequency depending on the parameters and geometry of the experiment [2].

The velocity fields during the reconfiguration events are needed to improve the understanding of the physical processes, however they are not provided in most studies. One reason is that the events occur on long time scales, which makes it difficult to capture reconfigurations with particle image velocimetry (PIV) while simultaneously resolving the relevant time scales of the large-scale circulation. Considering volumetric PIV, covering the complete time series until a reconfiguration event occurs is infeasible due to the associated extremely high computational requirements. Hence, measuring only during duration of an event would substantially reduce the amount of data and computational requirements. For the here considered case and parameter set, the waiting period between two events amounts to approximately $(140 \pm 70)$ min while the event duration is estimated to $(66 \pm 9)$ min. This is underlined in figure 1 showing temperature time series measured by four selected probes. From this it can be concluded that a suitable trigger could reduce the measurement time of a reconfiguration event by a factor of 3 .

Therefore, a system capable of automatically measuring the velocity fields during the reconfiguration events is required. Similar efforts to automate PIV are known to achieve spatial and/or parameterwise compositions on micro-scale [4] and wind-tunnel applications [5]. Further, the principle of starting PIV based on information obtained from separate flow measurements is described in the literature [6]. Such a flow-intrinsic trigger was applied by [7], who relied on measured density fluctuations to start the PIV.

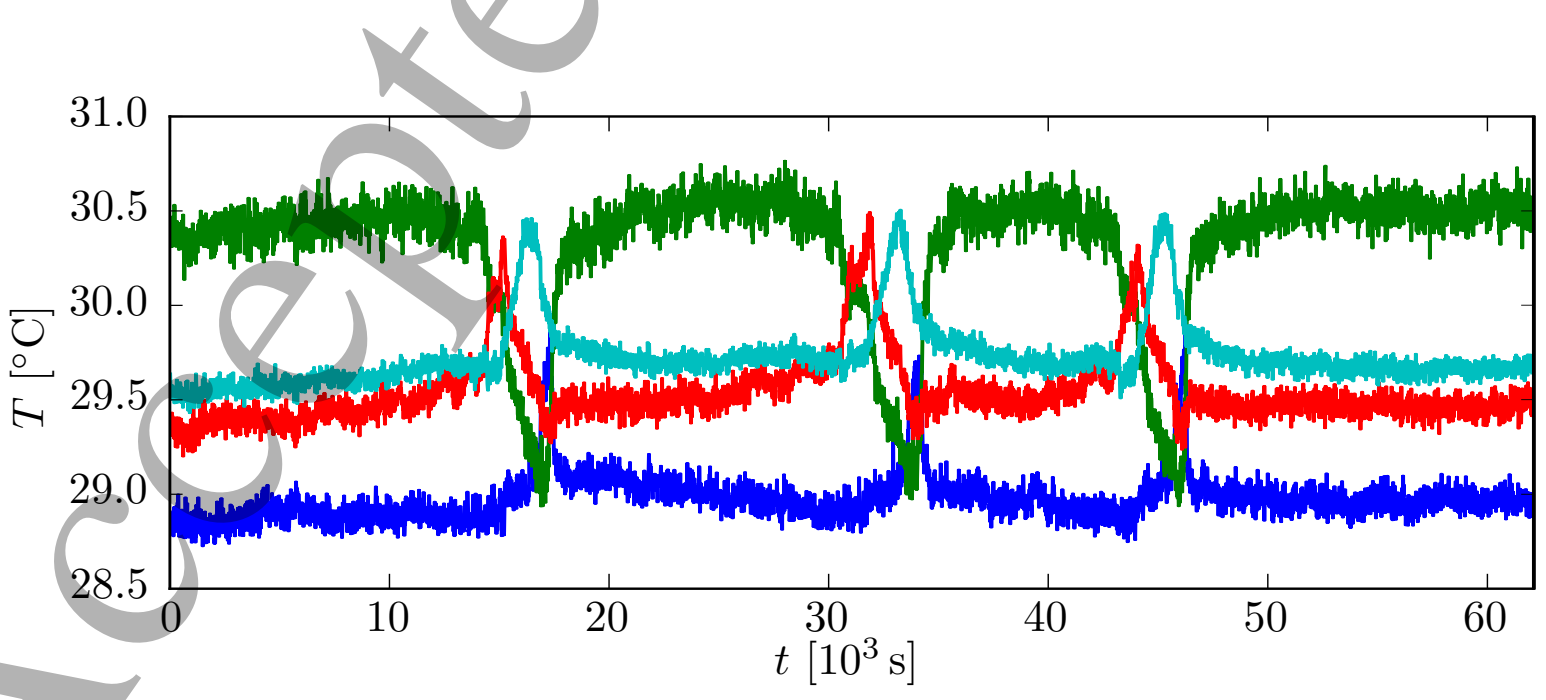

Figure 1. Temperature time series of four selected sensors in mixed convection flow exposing rare, non-periodic reconfiguration events. Details can be found in figure 4 . 
In a similar and more common approach, a conditional trigger is defined based on specific boundary conditions, for example externally induced pressure fluctuations [8]. For the here considered buoyancy-driven flows, it is well-known that footprints of reconfiguration events are visible in local temperature time series $[1,3]$. Thus, we developed the trigger condition based on the temperature signals explained in section 3.

The seeding with particles needed in PIV imposes an additional challenge for events occurring on long time scales. Since the large measurement volume of our convection sample, see section 2, requires tracer particles with strong light scattering capabilities, helium-filled soap bubbles (HFSB) $[9,10]$ are chosen. Yet, a suitable particle density must be provided at all times resulting in precipitation of bursted bubbles causing a soap film on the heated bottom plate of the convection sample, which influences the boundary conditions. This renders long continuous seeding periods and consequently a buffered PIV acquisition impossible. Thus, we propose a flow-intrinsic trigger for the seeding control which is developed to take these restrictions into account and is presented in section 2 in detail.

\section{Experimental setup}

In figure 2 a sketch of the mixed convection sample and the employed measurement systems is presented.

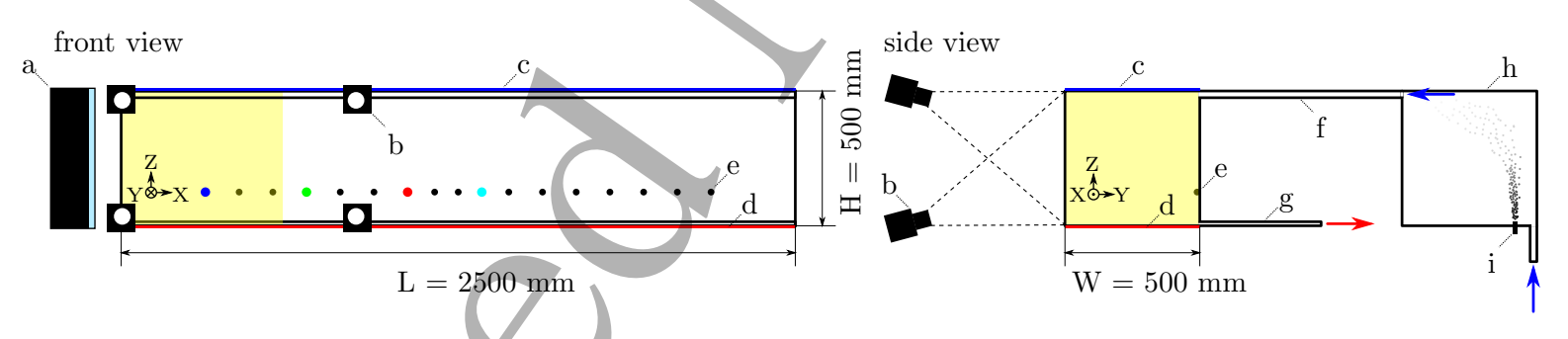

Figure 2. Experimental setup of the application case. The measurement domain is highlighted in yellow. Significant components are: a-LED light source, b-cameras, c-cooled plate, d-heated plate, e-temperature sensor array, f-inlet channel, g-outlet channel, h-seeding chamber, i-HFSB nozzle. The blue and red arrows indicate the forced convection.

The cuboidal sample is $L=2.5 \mathrm{~m}$ long and $H=W=0.5 \mathrm{~m}$ high and wide. It is comprised of an enclosure with a heated bottom plate (d) and a cooled top plate (c). Their temperature difference governs the Rayleigh number $R a$. Additionally, the $25 \mathrm{~mm}$ high and $750 \mathrm{~mm}$ long inlet channel (f) directs the forced flow into the sample. In figure 2, this flow is indicated by the blue arrows and leaves the sample (red arrow) through the $15 \mathrm{~mm}$ high and $450 \mathrm{~mm}$ long outlet channel (g). The volume flow and thus the mean inflow velocity $v_{i n}$ in the inlet is measured with a Venturi nozzle. For more details on the handling of the forced flow the reader is referred to [10]. The results presented in the following are obtained for $R a=1.4 \cdot 10^{8}$ and $R e=0.7 \cdot 10^{4}$, which is based on the sample height $H$ and the mean inflow velocity $v_{i n}$. The respective fluid 


\section{Flow-intrinsic trigger for PIV}

properties of air used for the calculations of these numbers are the kinematic viscosity $\nu=1.66 \cdot 10^{-5} \mathrm{~m}^{2} / \mathrm{s}$, the thermal diffusivity $\alpha=2.34 \cdot 10^{-5} \mathrm{~m}^{2} / \mathrm{s}$ and the thermal expansion coefficient $\beta=3.28 \cdot 10^{-3} 1 / \mathrm{K}$.

The temperatures $T(X, t)$ used for the trigger signal are measured with $17 \mathrm{Pt} 100$ temperature probes (e) installed as a linear array on the rear wall at a height protruding $10 \mathrm{~mm}$ into the sample.

The tomographic PIV setup consists of an LED array [11] as light source (a) and four cameras (b), which are positioned in a $Y$ distance of $1800 \mathrm{~mm}$ to the sample. The measurement domain is highlighted in yellow and spans $600 \mathrm{~mm}$ in $X$ direction while covering the complete cross-section. HFSB are introduced into the system in an upstream seeding chamber $(\mathrm{h})$ by a single nozzle (i), which is positioned next to the chamber inlet at $X \approx 280 \mathrm{~mm}$. Referring to [9], the nominal gaseous throughput of this nozzle type is in the range of $0.1 \%$ of the forced volume flow. Therefore, it can be assumed that the induced momentum is dispersed on the path to the sample. The unaffected state of the buoyancy-driven structures was furthermore checked by the means of the temperature signatures, as the eyent exposed to seeding showed no differences to unexposed events.

A time separation of $\tau=12 \mathrm{~ms}$ is chosen between the frames of the double-images, which exhibited a density of 0.005 particles per pixel (ppp). The latter results from the large particle images of the HFSB with a typical diameter of $6 \mathrm{px}$. The recorded double images are evaluated using a tomographic PIV routine, which is also described in $[10,12]$ and is based on a volume self calibration [13], a simultaneous multiplicative algebraic reconstruction technique (SMART) [14] and a multi-pass cross-correlation. Using the SMART, $1200 \times 1000 \times 1000$ voxels $(v x)$ are chosen for $(0.6 \times 0.5 \times 0.5) \mathrm{m}^{3}$ in physical space resulting in a voxel size of $s_{v x}=0.5 \frac{\mathrm{mm}}{\mathrm{vx}}$.

The interrogations volumes of the resulting $29 \times 24 \times 24$ vectors have an overlap of $50 \%$. By using the voxel particle displacement uncertainty of $\sigma_{\text {tomo }}=0.2 \mathrm{vx}$, which is typical for this setup [10], and the voxel size $s_{v x}$, the uncertainty of the velocities $\sigma_{v}=0.008 \mathrm{~m} / \mathrm{s}$ is estimated as follows.

Regarding the trigger, a block scheme of the information and control flow for the experiment is shown in figure 3. It depicts that the relevant output of the control system as well as all scalar quantities are logged on the experiment control computer to ensure temporal alignment. The starting trigger used by the control system is derived from the temperature measurements. When the trigger is released, respective control signals are spread via a transistor-transistor logic (TTL) interface. They are converted by the sequencing computer for cameras and illumination, while an amplifier and solenoid valves are used to control the seeding generator. This enables the interval seeding required for the measurement of rare flow events in seeding-sensitive systems. 


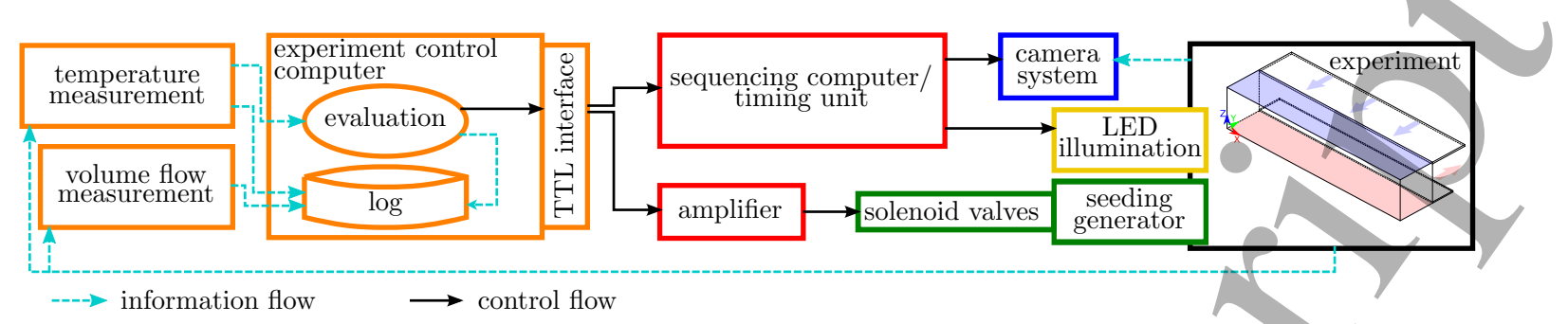

Figure 3. Block scheme of the information and control flow for an experiment with a conditional trigger.

\section{Trigger condition}

Earlier studies [1, 3] showed that flow reconfigurations manifest themselves as temperature changes. Vice versa, temperature changes can indicate the onset of the reconfigurations. To determine the actual trigger condition, preliminary tests for the regime of interest are necessary. If the relevant positions of the temperature sensors are not obvious, a temperature sensor array, which is not obstructing the subsequent velocity measurements, is recommended.

These tests are necessary to identify the characteristic temperature signature of the events. Using the latter, the sensors displaying the earliest indications of an event are chosen. In a last step, a trigger condition is defined with the main challenge to distinguish the onset of a reconfiguration from the temperature signal's fluctuations due to turbulence.

For the measurement presented in section 4, a straightforward intersection of two temperature time series $(2)$ was used as release condition with $\Delta t \approx 9$ s being one sampling interval of the temperature measurements.

$$
\begin{aligned}
& T(X=0.425 L, t-\Delta t)<T(X=0.275 L, t-\Delta t) \text { and } \\
& T(X=0.425 L, t) \quad \geq T(X=0.275 L, t)
\end{aligned}
$$

The PIV system is then activated for a given period of time on the first fulfillment of condition (2), since the eyent duration is consistent.

This condition was conservatively chosen to succeed in releasing the trigger for every reconfiguration event while completely avoiding false-positive releases of the trigger. Yet, this is achieved by a compromise, which includes a delayed release. A more sophisticated condition is proposed in section 5.

\section{Triggered PIV}

Figure 4 shows the temperature time series of selected sensors during the course of a reconfiguration. According to the approach discussed in section 3, (2) was used a trigger condition. Its fulfillment is marked by a yellow ring in figure 4. At this instant, 
the image acquisition and LED illumination, which operated at $0.9 \mathrm{~Hz}$, as well as the seeding generator are activated for the time span $\Delta t_{\mathrm{PIV}}=4000 \mathrm{~s}$, which equals the mean event duration. The standard deviation of the event duration is taken into account by considering the release delay of this condition. An additional delay $\Delta t_{\mathrm{HFSB}}$ must be considered for producing enough tracer particles and advecting them in all regions of the domain. This is a prerequisite for obtaining valid flow fields in tomographic PIV.

By applying the conditional trigger we captured three-dimensional velocity fields of a reconfiguration event in the mixed convection sample for the time span highlighted in yellow in figure 4. This time span equals approximately $75 \%$ of the event duration, which already covers a large extend of the structural dynamics of a reconfiguration.

Accordingly, figure 5 reflects four selected short-time averaged 3D velocity fields representing the flow at the time instants I-IV marked in figure 4 with velocity vectors in three cross-sections. In addition, the purple isosurfaces reflecting the absolute velocity value $|\mathbf{u}|=0.15 v_{\text {in }}$ are shown to visualize the convection roll's core. This allows to track the diagonal alignment of the observed roll part in the sample. The presented velocity fields are running averages of 9 frames to filter out turbulent velocity fluctuations.

Regarding the position of the roll core, we conclude that the observed roll part changes its orientation twice during a reconfiguration: First, the roll core is located at 
Flow-intrinsic trigger for PIV
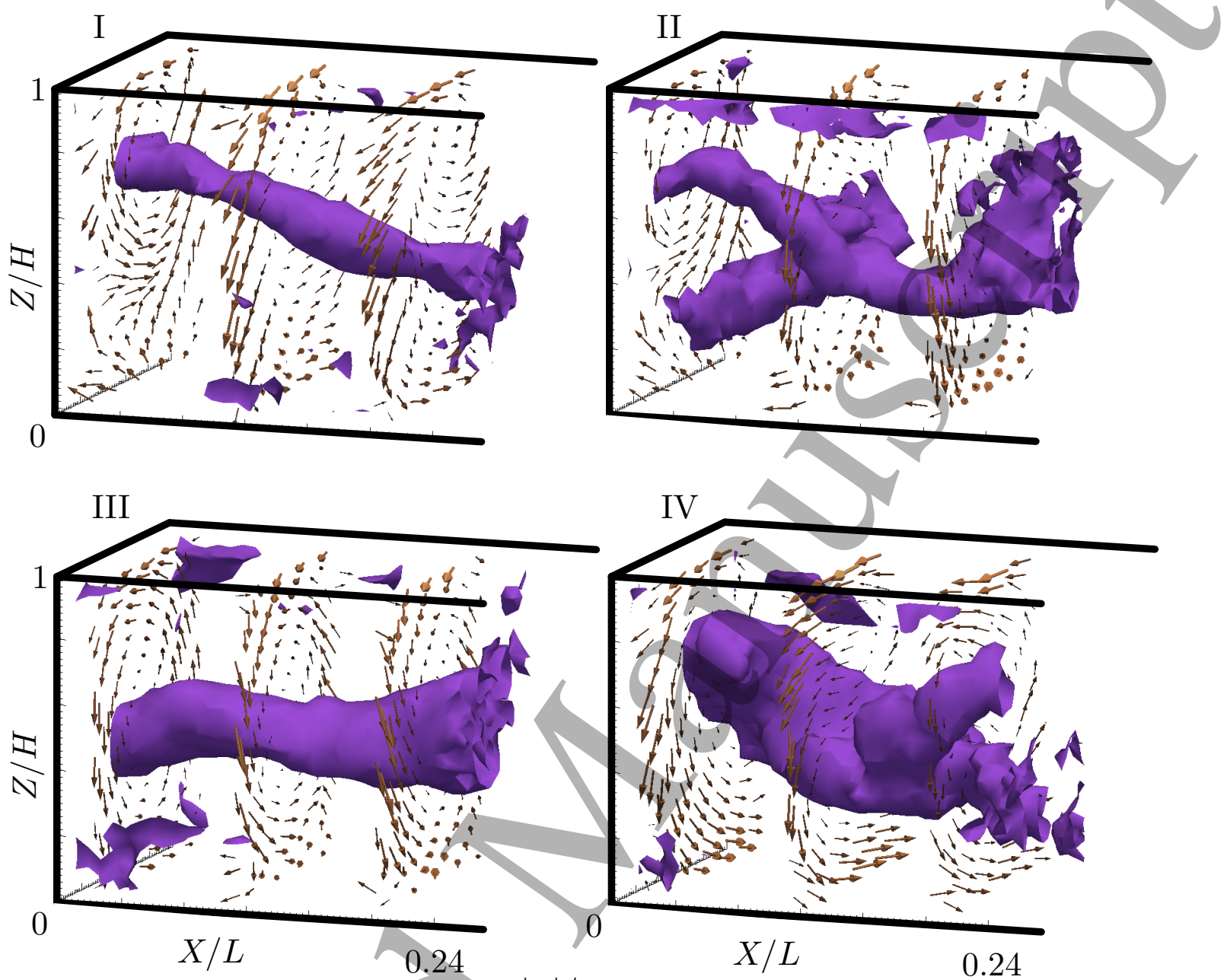

$|\mathbf{u}| / v_{\text {in }}$
IV

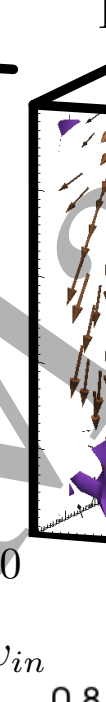

$0.4 \quad 0.8$

Figure 5. Exemplary flow fields displaying velocity vectors in three cross-sections and a velocity isosurface, which visualizes the convection roll center. The displayed flow fields are running averages of 9 frames. Their instants are marked in figure 4 .

an intermediate $Y$ position extending in $X$ direction at instant I. Next, the core aligns with a $X Y$ diagonal at II. This is followed by a realignment parallel to the $X$ axis at III before, at instant IV, the roll core aligns to the other $X Y$ diagonal, which is assumably its initial orientation before the event. The complete evolution of the velocity fields depicted in figure 5 is provided as animation in the supplementary material.

To our best knowledge, this is the first three-dimensional PIV measurement capturing a rare reconfiguration event in a buoyancy-driven flow. The presented trigger method/is a prerequisite to achieve this. 


\section{Flow-intrinsic trigger for PIV}

\section{Improved trigger condition}

The main shortcoming of the above presented trigger condition is its delayed release. For a more immediate release it is proposed to conduct the following analysis of the temperature series. From numerical investigations of Rayleigh-Bénard convection/15] relying on proper orthogonal decomposition (POD) it is known that reconfigurations can have significant precursors, which manifest themselves as intersections or sign changes in the time development coefficients of the most significant modes.

For the present case, POD, or more precisely, the here applied snapshot method [16] decomposes a $[S \times 1]$ temperature vector $\underline{T}(t)$ at a time $t$ with $S=17$ being the number of temperature sensors as follows:

$$
\underline{T}(t)=\Phi \underline{a}(t)
$$

In equation (3), $\Phi$ is the base of POD modes represented by an $[S \times N]$ matrix, where $N$ is the number of modes equaling the number of/time snapshots put into the POD. Further, the $\underline{a}(t)$ is a vector of size $[N \times 1]$ containing the time development coefficients for the respective time.

However, the POD base only reflects reconfigurations, if at least one completed event is available in the time series. To achieve an analysis for a still developing time series, the POD base $\Phi^{*}$ needs to be characterized in the first phase $\left(t^{*}\right)$ of the temperature measurement, as described by equation (4).

$$
\underline{T}\left(t^{*}\right)=\Phi^{*} \underline{a}\left(t^{*}\right)
$$

Since the POD modes are sorted by their eigenvalues, the temperatures can also be modeled by a reduced number of modes. Thus, it is valid to reduce $\Phi^{*}$ to an $[S \times M]$ matrix $\Phi_{\text {red }}^{*}$ with $M<S$.

Given that the flow parameters between the first and the triggered phase of the temperature measurement are not changed, the reduced base $\Phi_{\text {red }}^{*}$ is also a valid orthogonal base to represent the triggerd phase. According to this reduced base, a $[M \times 1]$ vector $\underline{b}(t)$ can be defined, which is comprised of the reconstructed coefficients for a time $t$ of the triggered time series, to substitute the time development coefficient of $(3)$.

$$
\underline{T}(t)=\Phi^{*} \underline{b}(t)
$$

This inverse problem [17], where $\underline{b}(t)$ is unknow, is expressed as an overdetermined linear system (5), due to $M<S$. However, the reconstructed coefficients in the linear system can be least-squares-fitted within fractions of a second. Hence, once the reduced base is computed from the first phase of the measurement, the reconstructed coefficients 
Figure 6. Time series of coefficients and temperatures in the time interval of the reconfiguration event shown in section 4: Top-Reconstructed coefficients $b_{n}$ using the POD base determined in a measurement with the same Ra and Re numbers. CenterTime development coefficients $a_{n}$ of the temperature series. Bottom-Temperature series used for the trigger described in section 3. The latter is highlighted with a yellow circle. The release instant for the improved condition is highlighted with a cyan circle.

which are also similar to time development coefficients can be calculated in real time in the lead up of the triggered PIV.

To test this approach, we generated a reduced base including the first five modes using a time interval reflecting reconfiguration events in the run up of the measurement presented in section 4. This interval exhibited the same dimensionless numbers as documented in section 2. Using this base, the reconstructed coefficients $b_{n}$, with $n$ being the mode number, are computed with the time series, which was used to trigger the PIV. Figure 6 renders the obtained reconstructed coefficients and, for comparison, the temperature series used for condition (2) and the time development coefficients of a POD using 17 temperature series. The time evolution of $a_{n}$ visible in the center plot is typical for this event type. Hence, it is possible to define a trigger condition after a POD is applied on the temperature series obtained in the first phase of the measurement. Figure 6 reflects that the coefficient $a_{3}$ rises during the onset of the event. In particular, mode 3 becomes the most dominant mode after passing $a_{1}$ substantially earlier than the trigger based on the raw temperatures signals. Figure 6 further reveals 


\section{Flow-intrinsic trigger for PIV}

the qualitative agreement of the development of the time development coefficients $a_{n}$ and the reconstructed coefficients $b_{n}$. From this we conclude that (6) can be used as an improved trigger condition.

$$
\begin{aligned}
& b_{3}(t-\Delta t)<b_{1}(t-\Delta t) \text { and } \\
& b_{3}(t) \quad \geq b_{1}(t)
\end{aligned}
$$

Similarly to (2), condition (6) is designed to activate the PIV system on the first fulfillment for a certain time. The improved condition would trigger approximately 30 min earlier than the trigger presented in section 3. This provides enough time for the seeding to be distributed in the complete volume of the sample, Thus, the onset of the reconfiguration event can be captured in the PIV. The reason for the earlier release of the POD based trigger is that the time development coefficients reflect the spatial distribution of the entire available temperature field. The latter is able to resolve smaller deviations with higher signal-to-noise ratios.

\section{Summary and outlook}

We presented a conditional trigger-based method to capture three-dimensional velocity fields of rare reconfiguration events in buoyancy-driven flows. The first key factor of this automated PIV approach is its control system, which activates the PIV equipment according to temperature signals measured within the flow. The second requirement is the LED-based illumination allowing for the long non-supervised standby periods before the measurement. It was shown that the developed method allows to record the changes of the flow structures during spontaneous reconfigurations.

In the future, the independent control of the seeding generator can be used to exactly control seeding densities by pulse-width modulation. Further potential lies in the trigger condition based on a POD of temperature series of a number of spatially distributed probes presented in section 5 , which will be applied to future measurements.

\section{Acknowledgments}

The authors thank Annika Köhne for her support provided by proofreading the manuscripts.

\section{References}

[1] Brown E and Ahlers G 2006 Rotations and cessations of the large-scale circulation in turbulent Rayleigh-Bénard convection Journal of Fluid Mechanics 568351

[2] Assaf M, Angheluta L and Goldenfeld N 2011 Rare Fluctuations and Large-Scale Circulation Cessations in Turbulent Convection Physical Review Letters 107(4) 044502

[3] Schmeling D, Bosbach J and Wagner C 2013 Oscillations of the large-scale circulation in turbulent mixed convection in a closed rectangular cavity Experiments in Fluids $\mathbf{5 4} 1517$ 
1

2

3

4

5

6

7

8

9

Flow-intrinsic trigger for PIV

[4] Augustsson P, Barnkob R, Wereley S T, Bruus H and Laurell T 2011 Automated and temperaturecontrolled micro-PIV measurements enabling long-term-stable microchannel acoustophoresis characterization Lab on a Chip 11(24) 4152-4164

[5] Michaux F, Mattern P and Kallweit S 2018 RoboPIV: how robotics enable PIV on a large industrial scale Measurement Science and Technology 29074009

[6] Adrian R J and Westerweel J 2010 Particle Image Velocimetry (Cambridge University Press)

[7] Woisetschläger J, Mayrhofer N, Lang H and Hampel B 2002 Experimental Investigation of Turbine Wake Flow by Interferometrically Triggered PIV and LDV Measurements ASME Turbo Expo 2002: Power for Land, Sea, and Air Volume 5: Turbo Expo 2002, Parts A and B pp 207-216

[8] Schröder A, Schanz D, Heine B and Dierksheide U 2013 Investigation of Transitional Flow Structures Downstream of a Backward-Facing-Step by Using 2D-2C-and High Resolution 3D3C- Tomo- PIV (Springer Berlin Heidelberg) pp 219-226

[9] Bosbach J, Kühn M and Wagner C 2008 Large scale particle image velocimetry with helium filled soap bubbles Experiments in Fluids 46 539-547

[10] Kühn M, Ehrenfried K, Bosbach J and Wagner C 2011 Large-scale tomographic particle image velocimetry using helium-filled soap bubbles Experiments in Fluids 50 929-948

[11] Willert C, Stasicki B, Klinner J and Moessner S 2010 Pulsed operation of high-power light emitting diodes for imaging flow velocimetry Measurement Science and Technology 21075402

[12] Kühn M, Ehrenfried K, Bosbach J and Wagner C 2012 Large-scale tomographic PIV in forced and mixed convection using a parallel SMART version Experiments in Fluids 53 91-103

[13] Wieneke B 2008 Volume self-calibration for 3D particle image velocimetry Experiments in Fluids $45549-556$

[14] Elsinga G E, Scarano F, Wieneke B and van Oudheusden B W 2006 Tomographic particle image velocimetry Experiments in Fluids 41 933-947

[15] Podvin B and Sergent A 2017 Precursor for wind reversal in a square Rayleigh-Bénard cell Physical Review E 95013112

[16] Sirovich L 1987 Turbulence and the dynamics of coherent structures. I - Coherent structures. II Symmetries and transformations. III - Dynamics and scaling Quarterly of Applied Mathematics 45 561-571

[17] Bui-thanh T, Damodaran M and Willcox K 2004 Aerodynamic data reconstruction and inverse design using proper orthogonal decomposition AIAA Journal 1505-1516 